\title{
Trends in Perioperative Venous Thromboembolism Associated with Major Noncardiac Surgery
}

\author{
Nathaniel R. Smilowitz ${ }^{1} \quad$ Navdeep Gupta $^{2} \quad$ Yu Guo $^{1} \quad$ Thomas S. Maldonado $^{3}$ John W. Eikelboom ${ }^{4}$ \\ Samuel Z. Goldhaber ${ }^{5}$ Sripal Bangalore ${ }^{1}$ Jeffrey S. Berger ${ }^{1,3}$ \\ ${ }^{1}$ Division of Cardiology, Department of Medicine, New York \\ University School of Medicine, New York, New York, United States \\ ${ }^{2}$ Department of Medicine, Medical College of Wisconsin, Milwaukee, \\ Wisconsin, United States \\ ${ }^{3}$ Division of Vascular Surgery, Department of Surgery, New York \\ University School of Medicine, New York, New York, United States \\ ${ }^{4}$ Department of Medicine, McMaster University, Hamilton, Ontario, \\ Canada \\ ${ }^{5}$ Division of Cardiovascular Medicine, Brigham and Women's \\ Hospital, Boston, Massachusetts, United States \\ TH Open 2017;1:e82-e91. \\ Address for correspondence Jeffrey Berger, MD, MS, Director of \\ Cardiovascular Thrombosis, Department of Medicine and Surgery, \\ Venous Thromboembolic Center, Marc and Ruti Bell Program in \\ Vascular Biology, New York University School of Medicine, 530 First \\ Avenue, Skirball 9R, New York, NY 10016, United States \\ (e-mail: jeffrey.berger@nyumc.org).
}

\author{
Abstract \\ Keywords \\ - deep vein thrombosis \\ - mortality \\ - perioperative \\ - pulmonary embolism \\ - surgery \\ - venous \\ thromboembolism
}

Background Venous thromboembolism (VTE) is a common vascular complication of noncardiac surgery.

Methods We evaluated national trends in perioperative in-hospital VTE incidence, management, and outcomes using a large database of hospital admissions from the United States. Patients aged $\geq 45$ years undergoing major noncardiac surgery from 2005 to 2013 were identified from the National Inpatient Sample. In-hospital perioperative VTE was defined as lower extremity deep vein thrombosis (DVT) or pulmonary embolism (PE), and the incidence was evaluated over time. Multivariable regression models with demographics and comorbidities as covariates were generated to estimate adjusted odds ratios (aOR).

Results Major noncardiac surgery was performed in 9,431,442 hospitalizations that met inclusion criteria, and perioperative VTE occurred in 99,776 patients $(1,057$ per 100,000 ), corresponding to an annual incidence of $\approx 53,000$ after applying sample weights. Over time, perioperative VTE per 100,000 surgeries increased by 135 ( $95 \%$ confidence interval [Cl]: 107-163), from 925 in 2005 to 1,060 in 2013 ( $p$ for trend $<0.001$; aOR [for 2013 vs. 2005]: $1.22,95 \% \mathrm{Cl}: 1.19-1.26$ ), due to increases in nonfatal VTE rates (from 840 [per 100,000 surgeries] in 2005 to 987 in 2013; p for trend $<0.001)$. Perioperative VTE occurred most frequently in patients undergoing thoracic $(2.0 \%)$ and vascular surgery (1.8\%). Mortality was higher in patients with VTE than those without VTE (aOR: 3.12, 95\% Cl: 3.05-3.20).

Conclusion Perioperative VTE occurs in approximately $1 \%$ of patients $\geq 45$ years undergoing major noncardiac surgery, with increasing incidence of nonfatal VTE over time.

\section{Introduction}

Venous thromboembolism (VTE), a composite of deep vein thrombosis (DVT) and pulmonary embolism (PE), is an important perioperative vascular complication associated with increased morbidity and mortality. As hospitalization for surgery is one of the strongest risk factors for VTE, clinical practice guidelines recommend routine prophylaxis for patients undergoing major general, abdominal, pelvic, orthopaedic, thoracic, or trauma surgery. ${ }^{1,2}$ However, data on the
DOI https://doi.org/ $10.1055 / \mathrm{s}-0037-1605360$ ISSN 2512-9465. (c) 2017 Georg Thieme Verlag KG Stuttgart · New York
License terms

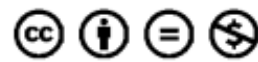


contemporary incidence, management, and outcomes of perioperative VTE after major noncardiac surgery remain limited. $^{3-7}$ We evaluated national trends in perioperative VTE incidence and outcomes associated with noncardiac surgery in a large administrative database of hospital admissions from the United States.

\section{Methods}

\section{Study Population}

The Healthcare Cost and Utilization Project's (HCUP) National Inpatient Sample (NIS) was used to identify patients undergoing major noncardiac surgery during the years 2005 to 2013. The HCUP NIS is a national administrative database of all discharge-level data collected from a $20 \%$ stratified sample of U.S. hospitals until 2012 and a 20\% stratified sample of discharges among all U.S. hospitals thereafter. Given the low prevalence of VTE in younger cohorts, patients 45 years or older with a principal International Classification of Diseases, Ninth Revision (ICD-9) procedure code for a major therapeutic operating room procedure (HCUP Procedure Class 4) were included in the analysis. Principal Clinical Classifications Software (CCS) procedure codes, aggregates of related primary ICD9 procedure codes by surgical subtype, were used to exclude patients who underwent cardiac procedures ( $n=1,465,792)$, cardiac surgery and cardiac transplantation ( $n=522,635)$, bone marrow transplantation $(n=16,934)$, ophthalmologic surgery $(n=11,458)$, radiation therapy $(n=8,216)$, dental surgery $(n=1,570)$, and nonoperating room procedures $(n=311) .{ }^{8}$ Patients with ICD-9 procedure codes for venous thrombectomy or embolectomy ( $n=1,389$ ) and inferior vena cava (IVC) filter placement in the primary position were also excluded $(n=133,446)$. Major noncardiac surgery CCS procedure codes were clustered into 12 major surgical subtypes for analysis: endocrine, general, genitourinary, gynecologic, neurosurgery, obstetrics, orthopaedic, otolaryngology, skin and breast, thoracic, noncardiac solid-organ transplant, and vascular surgery. Perioperative VTE diagnoses during the index surgical hospitalization were identified using ICD-9 diagnosis codes for acute PE $(415.1 \times)$ and lower extremity $\operatorname{DVT}(451.1 \times, 451.81,453.2,453.4 \times){ }^{4}$

\section{In-Hospital Management and Outcomes}

Infusion of thrombolytic agent (99.10) or pulmonary angiography and catheter-based thrombolysis (procedure codes 99.10 and 88.43) were defined based on ICD-9 procedure codes during inpatient hospitalization. ${ }^{9}$ IVC filter placement (38.7) was defined by an ICD-9 procedure code in a nonprimary position. The primary study outcome was in-hospital allcause mortality.

\section{Statistical Analysis}

Continuous variables were reported as mean \pm standard deviation (SD) and compared using the Student's $t$-test. Categorical variables were reported as percentages and compared by Chi-square tests. Analyses of proportions over time were performed using the Cochran-Armitage test for trend. Multivariable logistic regression models were generated to estimate odds ratios (aOR), adjusted for patient demographics, cardiovascular risk factors, and relevant comorbidities. Models included age, sex, race/ethnicity (as reported in the NIS), tobacco use, obesity, hypertension, hyperlipidemia, diabetes mellitus, chronic kidney disease, end-stage renal disease, coronary artery disease, peripheral arterial disease, congestive heart failure, valvular heart disease, prior VTE, chronic lung disease, alcohol abuse, malignancy, anemia, elective/urgent hospitalization, surgery type, and year of hospitalization as clinical covariates. When specified, HCUP NIS sampling weights were applied to extrapolate to nationwide findings. ${ }^{10}$ To facilitate data presentation in the tables, patient characteristics and outcomes were reported in 3-year intervals (2005-2007, 2008-2010, and 2011-2013) when specified. Statistical analyses were performed using SPSS 20 (IBM SPSS Statistics, Armonk, NY) and $\mathrm{R}$ ( $\mathrm{R}$ Foundation for Statistical Computing, Vienna, Austria). Statistical tests were two-sided and $p$-values $<0.05$ were considered to be statistically significant.

\section{Sensitivity Analyses}

Several sensitivity analyses were performed to validate the study findings. To exclude cases in which VTE may have preceded the primary noncardiac surgery, a sensitivity analysis was performed in the cohort of patients undergoing surgery within the first 48 hours of hospital admission. To account for declines in overall length of stay over time among patients hospitalized for surgery, rates of VTE per 100 inpatient hospital days were also determined.

\section{Results}

A total of 9,431,442 hospitalizations for major noncardiac surgery met the inclusion criteria (-supplementary Fig. S1, available online). This corresponds to an estimated 45,133,367 surgical hospitalizations in the United States during this time period, after applying sampling weights. VTE occurred in 99,776 patients ( 1,058 per 100,000 [1.06\%]) hospitalized for major noncardiac surgery, corresponding to an estimated 476,782 perioperative VTE events nationally after applying sampling weights. Among the patients with incident VTE, 57.1\% were diagnosed with DVT, 35.4\% with PE, and 9.5\% with DVT and PE. Demographics and baseline characteristics of patients with and without perioperative VTE are shown in - Table 1. Patients with VTE were older and more likely to be male. In a multivariable-adjusted model, major clinical predictors of perioperative VTE included advanced age, obesity, heart failure, malignancy, anemia, and a history of prior VTE (-Table 2).

\section{Trends in Perioperative VTE}

Over time, the number of perioperative VTE per 100,000 surgeries increased by 135 (95\% confidence interval [CI]: 107-163), from 925 in 2005 to 1,060 in 2013 ( $p$ for trend $<0.0001$; aOR: 1.22 , 95\% CI: 1.19-1.26). Similar trends were observed for DVT (608 per 100,000 surgeries in 2005 to 720 in 2013, $p$ for trend <0.0001) and PE (393 per 100,000 
e84 Venous Thromboembolism with Noncardiac Surgery Smilowitz et al.

Table 1 Baseline characteristics of patients undergoing major noncardiac surgery with and without in-hospital perioperative venous thromboembolism

\begin{tabular}{|c|c|c|c|c|}
\hline & $\begin{array}{l}\text { All surgeries } \\
(n=9,431,442)\end{array}$ & $\begin{array}{l}\text { Perioperative VTE } \\
(n=99,776)\end{array}$ & $\begin{array}{l}\text { No perioperative VTE } \\
(n=9,331,666)\end{array}$ & $\begin{array}{l}\text { Standardized } \\
\text { differences (\%) }\end{array}$ \\
\hline Age (mean $\pm S D$ ) & $65.64 \pm 12.25$ & $68.03 \pm 12.20$ & $65.62 \pm 12.25$ & 19.7 \\
\hline Female sex, no. (\%) & $5,317,479$ (56.5\%) & $51,681(51.8 \%)$ & $5,265,798(56.5 \%)$ & 9.5 \\
\hline \multicolumn{5}{|l|}{ Race/ethnicity } \\
\hline White non-Hispanic & $6,195,679(65.7 \%)$ & $62,929(63.1 \%)$ & $6,132,750(65.7 \%)$ & 5.5 \\
\hline Black non-Hispanic & 742,267 (7.9\%) & $11,213(11.2 \%)$ & $731,054(7.8 \%)$ & 11.6 \\
\hline Hispanic & $541,744(5.7 \%)$ & $5,501(5.5 \%)$ & $536,243(5.7 \%)$ & 1.0 \\
\hline Other race & $370,385(3.9 \%)$ & $3,663(3.7 \%)$ & 366,722 (3.9\%) & 1.4 \\
\hline Unknown & $1,581,367(16.8 \%)$ & $16,470(16.5 \%)$ & $1,564,897(16.8 \%)$ & 0.70 \\
\hline Obesity & $1,033,210(11.0 \%)$ & $12,004(12 \%)$ & $1,021,206(10.9 \%)$ & 3.4 \\
\hline Tobacco use & 972,930 (10.3\%) & $8,799(8.8 \%)$ & $964,131(10.3 \%)$ & 5.1 \\
\hline Hypertension & $5,530,310(58.6 \%)$ & $55,189(55.3 \%)$ & $5,475,121(58.7 \%)$ & 6.8 \\
\hline Hyperlipidemia & $2,788,252$ (29.6\%) & $24,560(24.6 \%)$ & 2,763,692 (29.6\%) & 11.3 \\
\hline Diabetes mellitus & $2,328,223(24.7 \%)$ & $24,479(24.5 \%)$ & $2,303,744(24.7 \%)$ & 0.40 \\
\hline Chronic kidney disease & $764,070(8.1 \%)$ & $11,970(12 \%)$ & $752,100(8.1 \%)$ & 13.1 \\
\hline End-stage renal disease & $253,005(2.7 \%)$ & $3,866(3.9 \%)$ & $249,139(2.7 \%)$ & 6.8 \\
\hline Coronary artery disease & $1,676,485(17.8 \%)$ & $16,802(16.8 \%)$ & $1,659,683(17.8 \%)$ & 2.5 \\
\hline Peripheral arterial disease & $669,582(7.1 \%)$ & $7,963(8 \%)$ & $661,619(7.1 \%)$ & 3.4 \\
\hline Valvular heart disease & $398,235(4.2 \%)$ & $4,824(4.8 \%)$ & $393,411(4.2 \%)$ & 3.0 \\
\hline History of heart failure & $604,658(6.4 \%)$ & $12,285(12.3 \%)$ & $592,373(6.3 \%)$ & 20.6 \\
\hline History of venous thromboembolism & $239,023(2.5 \%)$ & $5,342(5.4 \%)$ & $233,681(2.5 \%)$ & 14.7 \\
\hline Chronic pulmonary disease & $1,590,222(16.9 \%)$ & $19,510(19.6 \%)$ & $1,570,712(16.8 \%)$ & 7.1 \\
\hline Alcohol abuse & $200,432(2.1 \%)$ & $3,165(3.2 \%)$ & $197,267(2.1 \%)$ & 6.6 \\
\hline Malignancy & $495,945(5.3 \%)$ & $13,785(13.8 \%)$ & $482,160(5.2 \%)$ & 29.8 \\
\hline Anemia & $1,465,759(15.5 \%)$ & $27,104(27.2 \%)$ & $1,438,655(15.4 \%)$ & 29.0 \\
\hline Elective surgery & $5,770,943(61.4 \%)$ & $35,268(35.5 \%)$ & $5,735,675(61.6 \%)$ & 54.2 \\
\hline \multicolumn{5}{|l|}{ Surgery type } \\
\hline General & $2,039,973(21.6 \%)$ & $22,293(22.3 \%)$ & $2,017,680(21.6 \%)$ & 1.7 \\
\hline Endocrine & $110,200(1.2 \%)$ & $432(0.4 \%)$ & $109,768(1.2 \%)$ & 8.3 \\
\hline Genitourinary & $689,590(7.3 \%)$ & $6,517(6.5 \%)$ & $683,073(7.3 \%)$ & 3.1 \\
\hline Neurosurgery & $542,651(5.8 \%)$ & $7,343(7.4 \%)$ & $535,308(5.7 \%)$ & 6.6 \\
\hline Obstetric/gynecologic & $551,126(5.8 \%)$ & $2,215(2.2 \%)$ & $548,911(5.9 \%)$ & 18.7 \\
\hline Orthopaedic & $3,893,445(41.3 \%)$ & 34,839 (34.9\%) & $3,858,606(41.3 \%)$ & 13.3 \\
\hline Otolaryngology & $70,005(0.7 \%)$ & $429(0.4 \%)$ & $69,576(0.7 \%)$ & 4.1 \\
\hline Skin/breast & $407,787(4.3 \%)$ & $5,251(5.3 \%)$ & $402,536(4.3 \%)$ & 4.4 \\
\hline Thoracic & $212,572(2.3 \%)$ & $4,242(4.3 \%)$ & $208,330(2.2 \%)$ & 11.4 \\
\hline Transplant & $29,083(0.3 \%)$ & $374(0.4 \%)$ & $28,709(0.3 \%)$ & 1.2 \\
\hline Vascular & $885,010(9.4 \%)$ & 15,841 (15.9\%) & 869,169 (9.3\%) & 19.9 \\
\hline
\end{tabular}

Abbreviation: VTE, venous thromboembolism.

surgeries in 2005 to 462 in 2013, $p$ for trend $<0.0001$ ). Rates of fatal VTE per 100,000 surgeries declined over time, from 85 in 2005 to 73 in 2013 ( $p$ for trend $<0.0001$ ); nonfatal VTE per 100,000 surgeries increased from 840 in 2005 to 987 in 2013 ( $p$ for trend $<0.0001$ ). Trends in rates of perioperative
VTE are shown in - Table 3 and - Fig. 1A, B. VTE trends by sex and race are shown in -Supplementary Figs. S2 and S3 (available online). Men (vs. women) and non-Hispanic black patients (vs. other race/ethnicity groups) had higher rates of VTE at all time points ( $p<0.001$ for each time point). 
Table 2 Predictors of venous thromboembolism during hospitalization for major noncardiac surgery

\begin{tabular}{|c|c|c|}
\hline & $\begin{array}{l}\text { Adjusted odds ratio } \\
(95 \% \mathrm{Cl})\end{array}$ & p-Value \\
\hline Age (per year) & 1.01 (95\% Cl: $1.01-1.01)$ & $<0.001$ \\
\hline Female sex & 0.86 (95\% Cl: $0.85-0.87)$ & $<0.001$ \\
\hline \multicolumn{3}{|l|}{ Race/ethnicity } \\
\hline White non-Hispanic & Reference & \multirow{5}{*}{$<0.001$} \\
\hline Black non-Hispanic & 1.34 (95\% Cl: 1.31-1.37) & \\
\hline Hispanic & 0.95 (95\% Cl: $0.92-0.97)$ & \\
\hline Other race & 0.93 (95\% Cl: 0.9-0.96) & \\
\hline Unknown & 1.1 (95\% Cl: 1.08-1.12) & \\
\hline Obesity & 1.40 (95\% Cl: $1.37-1.43)$ & $<0.001$ \\
\hline Tobacco use & 0.82 (95\% Cl: $0.81-0.84)$ & $<0.001$ \\
\hline Hypertension & 0.79 (95\% Cl: 0.78-0.8) & $<0.001$ \\
\hline Hyperlipidemia & 0.85 (95\% Cl: $0.83-0.86)$ & $<0.001$ \\
\hline Diabetes mellitus & 0.88 (95\% Cl: 0.87-0.89) & $<0.001$ \\
\hline Chronic kidney disease & 1.02 (95\% Cl: 0.99-1.04) & 0.12 \\
\hline End-stage renal disease & 0.69 (95\% Cl: $0.66-0.72)$ & $<0.001$ \\
\hline Coronary artery disease & 0.84 (95\% Cl: $0.83-0.86)$ & $<0.001$ \\
\hline Peripheral arterial disease & 0.81 (95\% Cl: 0.79-0.83) & $<0.001$ \\
\hline Valvular heart disease & 0.94 (95\% Cl: $0.91-0.97)$ & $<0.001$ \\
\hline History of heart failure & 1.5 (95\% Cl: $1.47-1.53)$ & $<0.001$ \\
\hline $\begin{array}{l}\text { History of venous } \\
\text { thromboembolism }\end{array}$ & 2.05 (95\% Cl: 1.99-2.11) & $<0.001$ \\
\hline $\begin{array}{l}\text { Chronic pulmonary } \\
\text { disease }\end{array}$ & 1.04 (95\% Cl: $1.02-1.05)$ & $<0.001$ \\
\hline Alcohol abuse & 1.09 (95\% Cl: $1.05-1.13)$ & $<0.001$ \\
\hline Malignancy & 2.37 (95\% Cl: 2.33-2.42) & $<0.001$ \\
\hline Anemia & 1.62 (95\% Cl: 1.6-1.65) & $<0.001$ \\
\hline Elective surgery & 0.4 (95\% Cl: $0.4-0.41)$ & $<0.001$ \\
\hline \multicolumn{3}{|l|}{ Surgery type } \\
\hline General & Reference & \multirow[t]{12}{*}{$<0.001$} \\
\hline Endocrine & 0.64 (95\% Cl: 0.58-0.7) & \\
\hline Genitourinary & 1.06 (95\% Cl: $1.03-1.09)$ & \\
\hline Gynecologic & 0.71 (95\% Cl: $0.68-0.74)$ & \\
\hline Neurosurgery & 1.65 (95\% Cl: 1.61-1.7) & \\
\hline Obstetric & 0.14 (95\% Cl: $0.07-0.28)$ & \\
\hline Orthopaedic & 1.06 (95\% Cl: $1.04-1.08)$ & \\
\hline Otolaryngology & 0.63 (95\% Cl: $0.57-0.69)$ & \\
\hline Skin/breast & 1.29 (95\% Cl: $1.25-1.33)$ & \\
\hline Thoracic & 1.93 (95\% Cl: 1.86-1.99) & \\
\hline Transplant & 1.47 (95\% Cl: 1.32-1.63) & \\
\hline Vascular & 2.46 (95\% Cl: 2.4-2.51) & \\
\hline
\end{tabular}

Despite declines in overall length of stay for surgical admissions during the analysis period, rates of VTE per 100 inpatient hospital days increased over time, from 0.16 in 2005 to 0.21 in 2013 ( $p$ for trend $<0.001$ ). To exclude cases in which a VTE may have preceded an urgent surgery, a sensitivity analysis of patients undergoing noncardiac surgery within the first 48 hours of hospitalization was performed. The frequency of VTE (768 per 100,000 [0.77\%]) in this analysis was lower than the overall cohort, but exhibited similar trends (VTE: 677 per 100,000 surgeries in 2005 to 804 in 2013, p for trend $<0.0001$; DVT: 427 per 100,000 surgeries in 2005 to 525 in 2013, $p$ for trend $<0.0001$; PE: 302 per 100,000 surgeries in 2005 to 367 in 2013, $p$ for trend $<0.0001$ ).

\section{Surgery-Specific Risks}

In unadjusted analyses, patients undergoing thoracic surgery (2.0\%) and vascular surgery (1.8\%) had the highest absolute risks of perioperative VTE (-Fig. 2). In a model adjusted for demographics and clinical covariates, vascular surgery (aOR: 2.46; 95\% CI: 2.40-2.51) and thoracic surgery (aOR: 1.93; 95\% CI: 1.86-1.99) were the strongest independent predictors of perioperative VTE. Other surgical predictors of perioperative VTE after multivariable adjustment are shown in - Table 2.

\section{In-Hospital Management of VTE}

Hospital length of stay was longer for patients with perioperative VTE than those without VTE after noncardiac surgery (11 days; interquartile range [IQR]: 6-20] vs. 3 days [IQR: 2-6], $p<0.001)$. Length of stay for patients with perioperative VTE decreased slightly over time, from a median of 11 days (IQR: 7-19 days) in 2005 to 10 days (IQR: 6-18 days) in 2013 ( $p<0.0001$ ).

In-hospital placement of an IVC filter was performed in $24,014(24.1 \%)$ patients with perioperative VTE. IVC filters were placed in $41.0 \%$ of patients with DVT and PE, $24.7 \%$ of patients with DVT alone, and $18.1 \%$ of patients with PE alone. Among patients with perioperative PE, systemic thrombolytic therapy was administered in $3.3 \%$ of cases and catheterdirected thrombolysis was performed in $0.3 \%$ of cases.

\section{Outcomes of Perioperative VTE}

In-hospital mortality was higher in patients with perioperative VTE versus those without VTE in unadjusted analyses (8.2 vs. $1.5 \%, p<0.001$ ) and after adjustment for clinical covariates (aOR: 3.12, 95\% CI: 3.05-3.20). Increased odds of mortality after multivariable adjustment were observed for patients with perioperative PE versus those without PE (aOR: 4.96, 95\% CI: 4.80-5.13 for PE) and perioperative DVT versus those without DVT (aOR: 2.10, 95\% CI: 2.03-2.18). Among patients with perioperative VTE, mortality was significantly higher for patients with PE than patients with DVT (11.2 vs. $6.4 \%, p<0.001$ ).

In-hospital mortality associated with perioperative VTE declined from $9.2 \%$ in 2005 to $6.9 \%$ in 2013 ( $p$ for trend $<0.0001$; aOR: 0.88, 95\% CI: 0.84-0.93; - Fig. 3). Adjusted mortality decreased significantly between 2005 and 2013 among patients with PE (aOR: 0.84; 95\% CI: 0.72-0.97). In contrast, adjusted mortality did not change significantly among patients with DVT (aOR: 0.93; 95\% CI: 0.80-1.08; - Fig. 4).

\section{Discussion}

Among 9.4 million U.S. hospitalizations for major noncardiac surgery from 2005 to 2013, perioperative VTE occurred in $1.0 \%$ of cases, and rates increased over time. Major predictors 
Table 3 Trends in baseline characteristics of all patients undergoing major noncardiac surgery and the frequency of in-hospital perioperative venous thromboembolic events over time

\begin{tabular}{|c|c|c|c|c|}
\hline & $\begin{array}{l}2005-2007 \\
(n=3,109,729)\end{array}$ & $\begin{array}{l}2008-2010 \\
(n=3,263,943)\end{array}$ & $\begin{array}{l}2011-2013 \\
(n=3,057,770)\end{array}$ & $p$-Value \\
\hline Age (mean $\pm S D)$ & $65.74 \pm 12.53$ & $65.51 \pm 12.30$ & $65.69 \pm 11.92$ & $<0.0001$ \\
\hline Female & $1,779,263(57.4 \%)$ & $1,838,944(56.5 \%)$ & $1,699,272(55.6 \%)$ & $<0.0001$ \\
\hline Elective surgery & $1,894,112(61.1 \%)$ & $1,997,551(61.3 \%)$ & $1,879,280(61.7 \%)$ & \\
\hline \multicolumn{5}{|l|}{ Surgery type } \\
\hline General & $671,964(21.6 \%)$ & $707,741(21.7 \%)$ & $660,268(21.6 \%)$ & \multirow[t]{12}{*}{$<0.0001$} \\
\hline Endocrine & $38,351(1.2 \%)$ & $39,934(1.2 \%)$ & $31,915(1 \%)$ & \\
\hline Genitourinary & $239,280(7.7 \%)$ & $244,593(7.5 \%)$ & 205,717 (6.7\%) & \\
\hline Gynecologic & $224,192(7.2 \%)$ & 191,069 (5.9\%) & $128,154(4.2 \%)$ & \\
\hline Neurosurgery & $184,159(5.9 \%)$ & $184,416(5.7 \%)$ & $174,076(5.7 \%)$ & \\
\hline Obstetric & $2,665(0.1 \%)$ & $2,671(0.1 \%)$ & $2,375(0.1 \%)$ & \\
\hline Orthopedic & $1,171,541(37.7 \%)$ & $1,348,043(41.3 \%)$ & $1,373,861(44.9 \%)$ & \\
\hline Otolaryngology & $24,600(0.8 \%)$ & $23,422(0.7 \%)$ & $21,983(0.7 \%)$ & \\
\hline Skin/breast & $162,165(5.2 \%)$ & $133,012(4.1 \%)$ & $112,610(3.7 \%)$ & \\
\hline Thoracic & $67,700(2.2 \%)$ & $75,917(2.3 \%)$ & $68,955(2.3 \%)$ & \\
\hline Transplant & $7,994(0.3 \%)$ & $11,406(0.3 \%)$ & $9,683(0.3 \%)$ & \\
\hline Vascular & $315,118(10.1 \%)$ & $301,719(9.2 \%)$ & $268,173(8.8 \%)$ & \\
\hline \multicolumn{5}{|l|}{ Adverse cardiovascular events } \\
\hline Pulmonary embolism & $12,842(0.41 \%)$ & $15,558(0.48 \%)$ & $14,404(0.47 \%)$ & $<0.0001$ \\
\hline Deep vein thrombosis & $20,259(0.65 \%)$ & 24,077 (0.74\%) & $22,076(0.72 \%)$ & $<0.0001$ \\
\hline Venous thromboembolism (VTE) & $30,530(1.0 \%)$ & $36,227(1.1 \%)$ & $33,019(1.1 \%)$ & $<0.0001$ \\
\hline Fatal VTE & $2,752(9 \%)$ & $3,006(8.3 \%)$ & $2,443(7.4 \%)$ & $<0.0001$ \\
\hline Nonfatal VTE & $27,778(91 \%)$ & $33,221(91.7 \%)$ & $30,576(92.6 \%)$ & $<0.0001$ \\
\hline Death & $54,701(1.8 \%)$ & $51,889(1.6 \%)$ & $42,076(1.4 \%)$ & $<0.0001$ \\
\hline
\end{tabular}

included advancing age, male sex, obesity, heart failure, malignancy, anemia, and a history of prior VTE. In adjusted analyses, patients undergoing thoracic and noncardiac vascular surgery had the highest risks of perioperative VTE.

The observed increase in the incidence of perioperative VTE occurred over time despite a renewed focus on the importance of perioperative VTE prophylaxis as an unmet need and public health crisis. ${ }^{11}$ Risk assessment models to predict VTE risk have been developed and validated in multiple surgical subspecialties, ${ }^{12-16}$ thereby enabling providers to identify patients who might derive the greatest benefit from appropriate VTE thromboprophylaxis. There is evidence that providers have increased adherence to perioperative VTE prophylaxis over time. Among patients undergoing colorectal surgery, for example, the rate of perioperative pharmacological thromboprophylaxis increased substantially from $31.6 \%$ in 2006 to $86.4 \%$ in 2011 . However, there is conflicting evidence regarding associations between increased rates of pharmacological thromboprophylaxis and declines in perioperative VTE. ${ }^{14,17,18}$ Data on in-hospital pharmacological thromboprophylaxis were not available from the NIS dataset; so, its impact on VTE incidence cannot be assessed in the present study. However, based on the trends reported from individual surgical subspecialties, it is possible that rates of perioperative pharmacological thromboprophylaxis increased nationally during the analysis period. ${ }^{17}$

The etiology of increasing rates of VTE may also be linked to changes in approaches to clinical diagnosis. With increasing awareness of perioperative VTE risk, novel risk scores and prediction rules, and high-resolution multidetector $\mathrm{CT}$ angiography for PE, increased diagnostic sensitivity may be at least partially responsible for the increased incidence of perioperative VTE over time. In fact, a rising incidence of VTE has also been reported among nonsurgical hospitalized patients. ${ }^{19}$ Finally, the observed increases in VTE incidence may be related to changes in burden of risk factors for venous thromboembolic disease, or increasing surgical case complexity over time.

In this analysis, mortality associated with perioperative VTE declined over time. This finding is consistent with trends described in nonsurgical and surgical subspecialty populations with acute VTE. ${ }^{4,20-23}$ In a recent report from the Computerized Registry of Patients with Venous Thromboembolism (RIETE), a multinational, observational cohort study of patients with any diagnosis of VTE, risk-adjusted all-cause mortality rates associated with acute PE declined substantially between 2001 and 


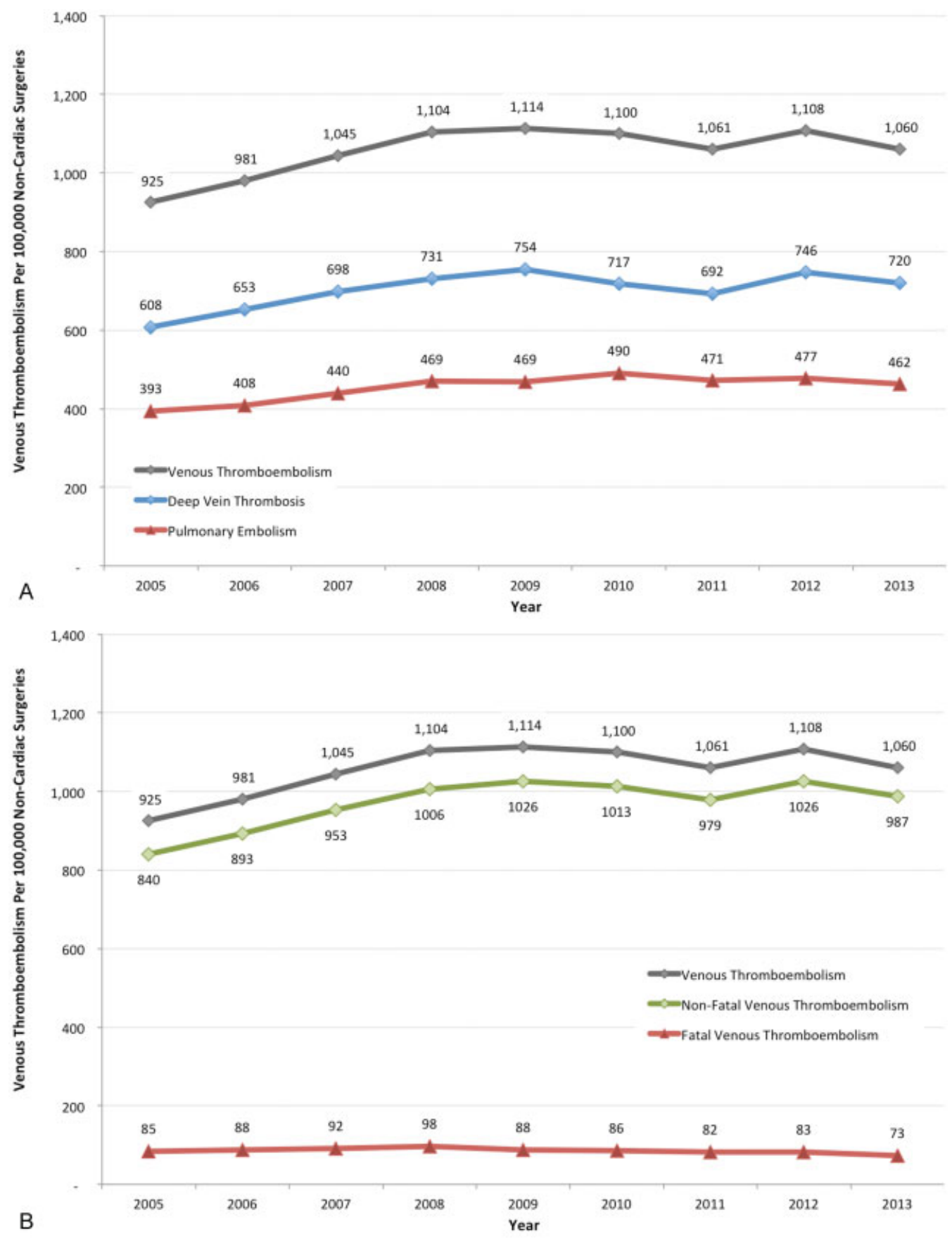

Fig. 1 (A) Trends in rates of perioperative venous thromboembolism from 2005 to 2013. $p$-Value for trend $<0.001$. (B) Trends in rates of perioperative fatal and nonfatal venous thromboembolism over time, from 2005 to 2013. $p$-Value for trend $<0.001$.

2013. ${ }^{21}$ There was an increased use of low-molecular-weight heparins, thrombolytic therapy, and surgical embolectomy over time. It is conceivable that similar trends occurred in the perioperative population, and that these changes in clinical management may have contributed to improved outcomes. Alternatively, improved VTE case fatality may be related to enhanced diagnostic sensitivity for VTE and identification of lower risk patients, thereby leading to a selection bias in case fatality over time. ${ }^{23}$ Changes in the diagnostic sensitivity of thoracic imaging may be related to the observed declines in mortality among patients with perioperative PE, but not peri- operative DVT. Changing patient's risk profiles over time might also contribute to the observed declines in perioperative VTE mortality, but these trends persisted even after adjustment for baseline demographics and relevant comorbidities.

Vena cava filter placement was performed in $24.1 \%$ of patients with VTE undergoing noncardiac surgery, despite little data to support the efficacy of filter placement for VTE and significant inter-hospital variation reported in practice. ${ }^{24-26}$ In the present analysis, catheter-directed and systemic thrombolytic therapies were infrequently used in the setting of noncardiac surgery. This is likely related to the anticipated risks of 


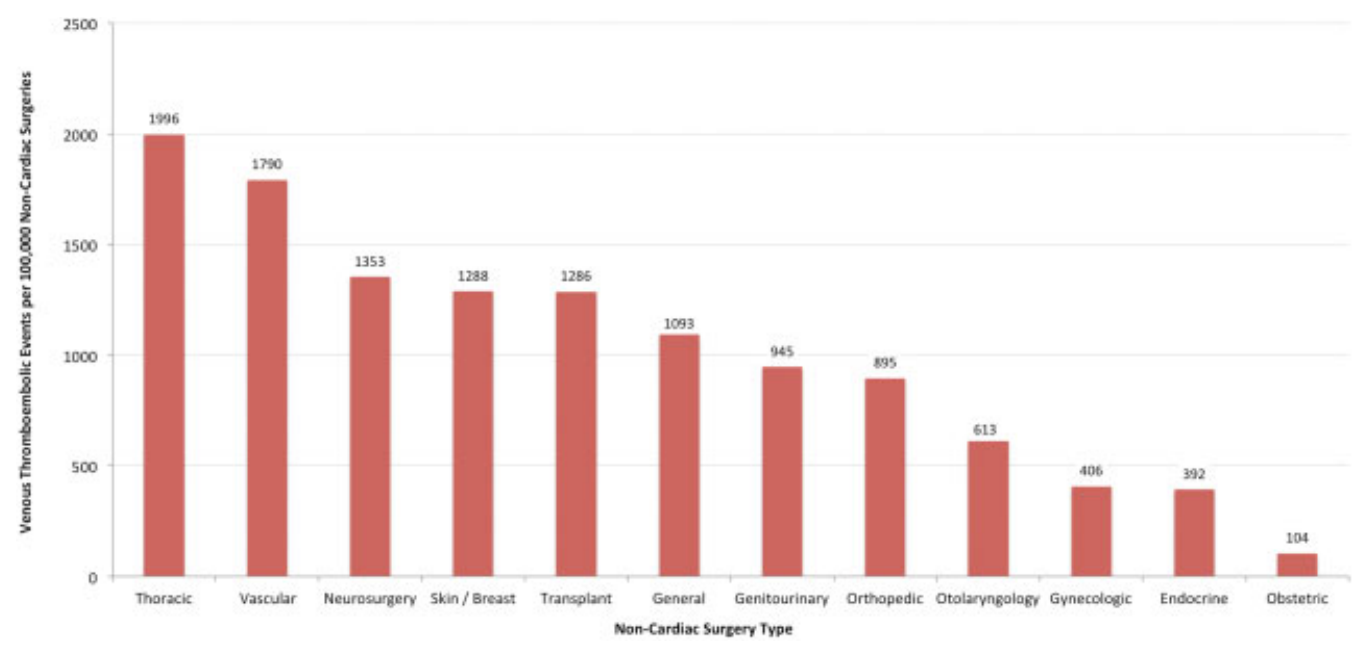

Fig. 2 Frequency of perioperative venous thromboembolism by type of major noncardiac surgery.

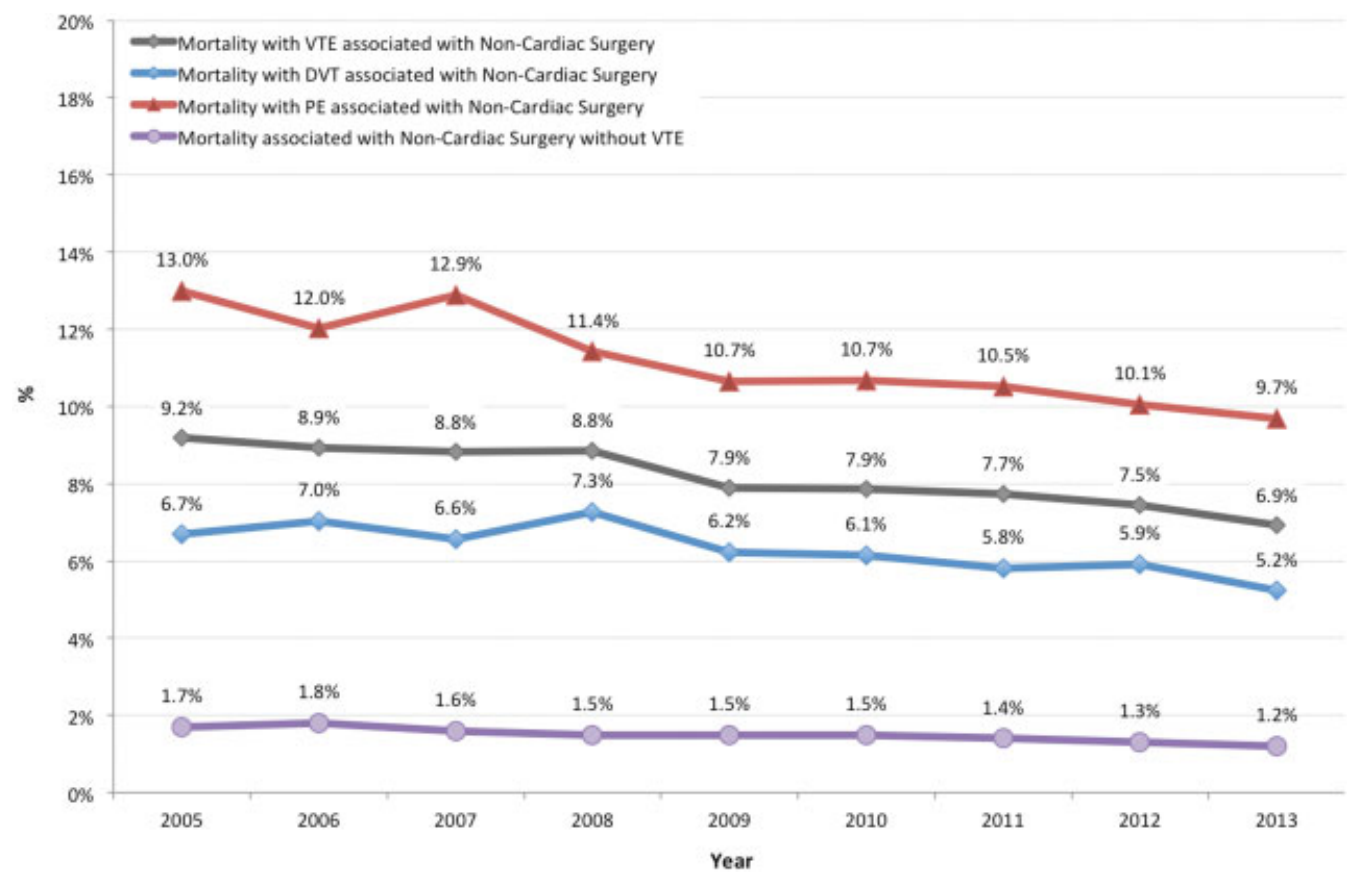

Fig. 3 In-hospital mortality trends among patients with perioperative venous thromboembolism. $p$-Value for trend $<0.001$.

surgical site bleeding associated with thrombolytic therapies in this context.

There are several limitations of this analysis. First, the data are derived from diagnosis and procedure codes recorded in a large nationwide administrative database of inpatient hospitalizations. As a consequence, data may be subject to bias, errors in reporting and coding, and the diagnosis of VTE may be underreported. As the NIS captures only in-hospital administrative data, it may underestimate the true incidence of perioperative VTE, since more than one-third of postoperative VTEs occur postdischarge. ${ }^{7,27-30}$ A particularly vulnerable period is the first month after hospital discharge, when pharmacological thromboprophylaxis is often not utilized. Similarly, deaths that occurred after hospital discharge were not recorded. Further- more, the NIS database does not include patients undergoing ambulatory or same-day surgery, and risks of VTE in this setting could not be determined. Second, the present analysis was limited to adults aged $\geq 45$, a population enriched for cardiovascular events and at the greatest risk for VTE. ${ }^{31}$ Trends in perioperative VTE were not determined for younger, low-risk patient groups. Third, data on in-hospital and discharge medical management were not available from the NIS database, and use of antiplatelet agents, unfractionated and low-molecularweight heparins, vitamin $\mathrm{K}$ antagonists, and direct oral anticoagulants for VTE prophylaxis and treatment could not be determined. The absence of data on rates of VTE prophylaxis during the study period limits the interpretation of VTE trends. Similarly, bleeding complications associated with VTE 

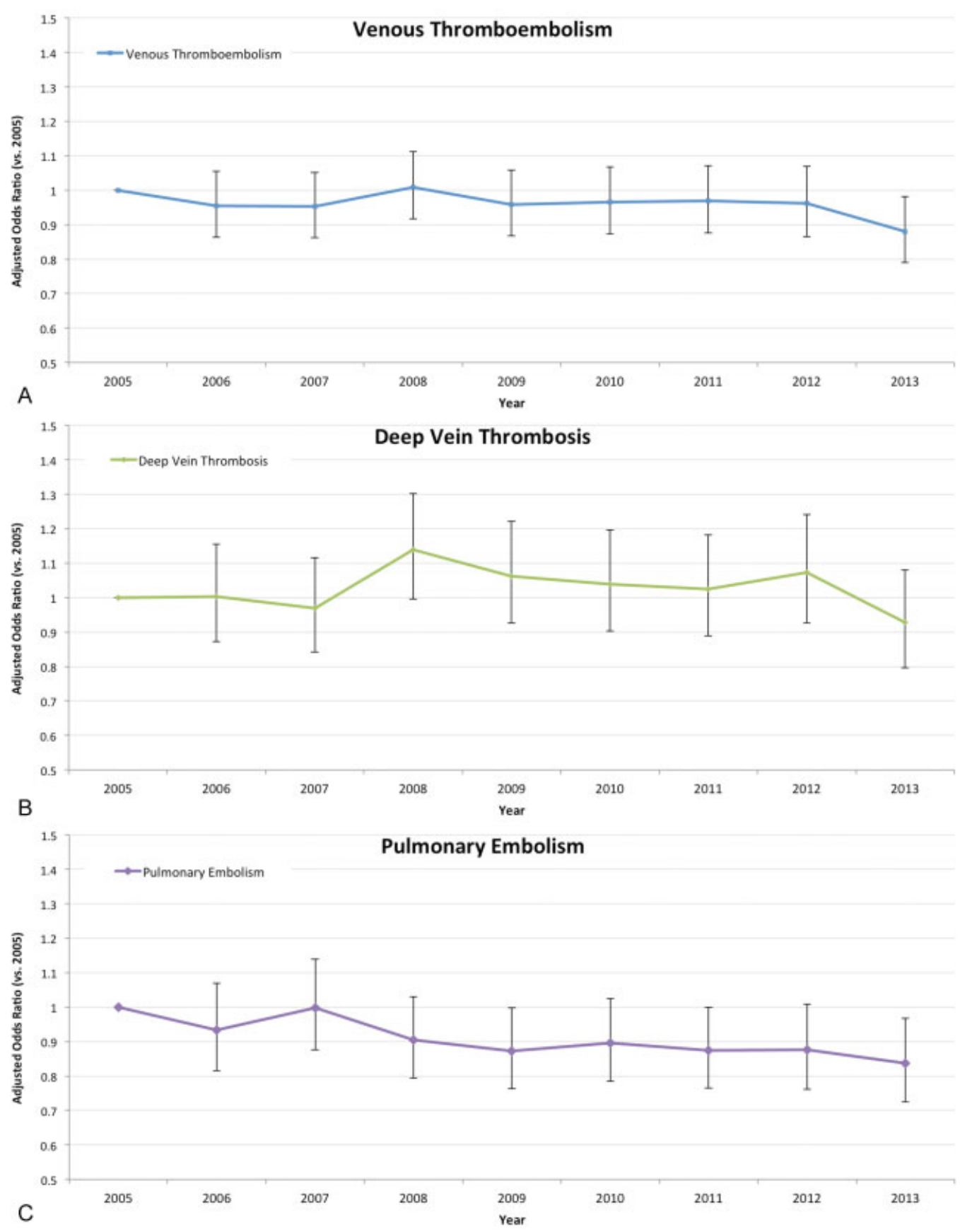

Fig. 4 Adjusted odds ratio(OR) for in-hospital mortality associated with venous thromboembolism (A), deep vein thrombosis (B), and pulmonary embolism (C) after noncardiac surgery, according to year. Models were adjusted for patient demographic characteristics, cardiovascular risk factors, and surgical type. Adjusted ORs are calculated with 2005 as the reference year. Bars represent 95\% confidence intervals.

prophylaxis and treatment could not be determined. Fourth, the sequence of surgery preceding VTE cannot be definitively established in this administrative database. However, patients with primary procedure codes for surgeries intended to treat VTE, such as venous thrombectomy and embolectomy, were excluded from the analysis. Furthermore, patients would be unlikely to undergo major nonvascular, noncardiac surgery in the setting of a known acute DVT or PE in most cases.

In conclusion, this is the largest analysis of in-hospital perioperative VTE in patients undergoing major noncardiac surgery in the United States. Perioperative VTE occurred in approximately $1 \%$ of patients undergoing major noncardiac surgery, with an increasing incidence over time. In-hospital mortality was significantly higher among patients with perioperative VTE in comparison to those without VTE. Overall perioperative VTE case fatality rates declined between 2005 and 2013, with significant decreases observed among patients with perioperative PE, but not DVT. Additional measures to prevent and improve management of perioperative VTE are warranted. 


\section{Conflict of Interest}

Dr. Eikelboom receives consulting fees and/or honoraria from Astra-Zeneca, Bayer Boehringer-Ingelheim, BristolMyer-Squibb, Daiichi-Sankyo, Eli-Lilly, Glaxo-Smith-Kline, Pfizer, Janssen, and Sanofi-Aventis. Dr. Eikelboom also receives grants and/or in-kind support from Astra-Zeneca, Bayer, Boehringer-Ingelheim, Bristol-Myer-Squibb, GlaxoSmith-Kline, Pfizer, Janssen, and Sanofi-Aventis. Dr. Goldhaber receives research support from BiO2 Medical, Boehringer-Ingelheim, BMS, BTG EKOS, Daiichi, Janssen, NHLBI, and the Thrombosis Research Institute. Dr. Goldhaber serves as a consultant for Agile, Bayer, Boehringer-Ingelheim, BMS, Daiichi, Janssen, Portola, and Zafgen. The remaining authors report no relationships that could be construed as a conflict of interest.

All authors had access to the data and a role in writing the manuscript.

\section{Funding}

Dr. Berger was partially funded by the NHLBI of the NIH (HL114978).

\section{References}

1 Falck-Ytter Y, Francis CW, Johanson NA, et al. Prevention of VTE in orthopedic surgery patients: Antithrombotic Therapy and Prevention of Thrombosis, 9th ed: American College of Chest Physicians Evidence-Based Clinical Practice Guidelines. Chest 2012; 141(2, Suppl):e278S-e325S

2 Gould MK, Garcia DA, Wren SM, et al. Prevention of VTE in nonorthopedic surgical patients: Antithrombotic Therapy and Prevention of Thrombosis, 9th ed: American College of Chest Physicians Evidence-Based Clinical Practice Guidelines. Chest 2012;141(2, Suppl):e227S-e277S

3 White RH, Zhou H, Romano PS. Incidence of symptomatic venous thromboembolism after different elective or urgent surgical procedures. Thromb Haemost 2003;90(03):446-455

4 Trinh VQ, Karakiewicz PI, Sammon J, et al. Venous thromboembolism after major cancer surgery: temporal trends and patterns of care. JAMA Surg 2014;149(01):43-49

5 Assareh H, Chen J, Ou L, Hollis SJ, Hillman K, Flabouris A. Rate of venous thromboembolism among surgical patients in Australian hospitals: a multicentre retrospective cohort study. BMJ Open 2014;4(10):e005502

6 Yhim HY, Jang MJ, Bang SM, et al. Incidence of venous thromboembolism following major surgery in Korea: from the Health Insurance Review and Assessment Service database. J Thromb Haemost 2014;12(07):1035-1043

7 Sweetland S, Green J, Liu B, et al; Million Women Study collaborators. Duration and magnitude of the postoperative risk of venous thromboembolism in middle aged women: prospective cohort study. BMJ 2009;339:b4583

8 Smilowitz NR, Gupta N, Ramakrishna H, Guo Y, Berger JS, Bangalore S. Perioperative major adverse cardiovascular and cerebrovascular events associated with noncardiac surgery. JAMA Cardiol 2017;2(02):181-187

9 Patel N, Patel NJ, Agnihotri K, et al. Utilization of catheter-directed thrombolysis in pulmonary embolism and outcome difference between systemic thrombolysis and catheter-directed thrombolysis. Catheter Cardiovasc Interv 2015;86(07):1219-1227

10 HCUP. Trend Weights for HCUP NIS Data. Rockville, MD: Agency for Healthcare Research and Quality; September 2014. Available at: http://www.hcup-us.ahrq.gov/db/nation/nis/trendwghts.jsp. Accessed May 1, 2016

11 Office of the Surgeon General (US); National Heart, Lung, and Blood Institute (US). The surgeon general's call to action to prevent deep vein thrombosis and pulmonary embolism. Publications and Reports of the Surgeon General. Rockville, MD; 2008

12 Caprini JA. Thrombosis risk assessment as a guide to quality patient care. Dis Mon 2005;51(2-3):70-78

13 Bahl V, Hu HM, Henke PK, Wakefield TW, Campbell DA Jr, Caprini JA. A validation study of a retrospective venous thromboembolism risk scoring method. Ann Surg 2010;251(02):344-350

14 Cassidy MR, Rosenkranz P, McAneny D. Reducing postoperative venous thromboembolism complications with a standardized risk-stratified prophylaxis protocol and mobilization program. J Am Coll Surg 2014;218(06):1095-1104

15 Pannucci CJ, Laird S, Dimick JB, Campbell DA, Henke PK. A validated risk model to predict 90-day VTE events in postsurgical patients. Chest 2014;145(03):567-573

16 Rogers SO Jr, Kilaru RK, Hosokawa P, Henderson WG, Zinner MJ, Khuri SF. Multivariable predictors of postoperative venous thromboembolic events after general and vascular surgery: results from the patient safety in surgery study. J Am Coll Surg 2007; 204(06):1211-1221

17 Nelson DW, Simianu VV, Bastawrous AL, et al; Colorectal Writing Group for Surgical Care and Outcomes Assessment Program-Comparative Effectiveness Research Translation Network (SCOAP-CERTAIN) Collaborative. Thromboembolic complications and prophylaxis patterns in colorectal surgery. JAMA Surg 2015;150(08):712-720

18 Johnbull EA, Lau BD, Schneider EB, Streiff MB, Haut ER. No association between hospital-reported perioperative venous thromboembolism prophylaxis and outcome rates in publicly reported data. JAMA Surg 2014;149(04):400-401

19 Huang W, Goldberg RJ, Anderson FA, Kiefe CI, Spencer FA. Secular trends in occurrence of acute venous thromboembolism: the Worcester VTE study (1985-2009). Am J Med 2014;127(09):829-39.e5

20 Bikdeli B, Wang Y, Minges KE, et al. Vena caval filter utilization and outcomes in pulmonary embolism: Medicare hospitalizations from 1999 to 2010. J Am Coll Cardiol 2016;67(09):1027-1035

21 Jiménez D, de Miguel-Díez J, Guijarro R, et al; RIETE Investigators. Trends in the management and outcomes of acute pulmonary embolism: analysis from the RIETE Registry. J Am Coll Cardiol 2016;67(02):162-170

22 Park B, Messina L, Dargon P, Huang W, Ciocca R, Anderson FA. Recent trends in clinical outcomes and resource utilization for pulmonary embolism in the United States: findings from the nationwide inpatient sample. Chest 2009;136(04):983-990

23 Wiener RS, Schwartz LM, Woloshin S. Time trends in pulmonary embolism in the United States: evidence of overdiagnosis. Arch Intern Med 2011;171(09):831-837

24 White RH, Brunson A, Romano PS, Li Z, Wun T. Outcomes after vena cava filter use in noncancer patients with acute venous thromboembolism: a population-based study. Circulation 2016; 133(21):2018-2029

25 Muriel A, Jiménez D, Aujesky D, et al; RIETE Investigators. Survival effects of inferior vena cava filter in patients with acute symptomatic venous thromboembolism and a significant bleeding risk. J Am Coll Cardiol 2014;63(16):1675-1683

26 White RH, Geraghty EM, Brunson A, et al. High variation between hospitals in vena cava filter use for venous thromboembolism. JAMA Intern Med 2013;173(07):506-512

27 Merkow RP, Bilimoria KY, McCarter MD, et al. Post-discharge venous thromboembolism after cancer surgery: extending the case for extended prophylaxis. Ann Surg 2011;254(01):131-137

28 Nelson RE, Grosse SD, Waitzman NJ, et al. Using multiple sources of data for surveillance of postoperative venous thromboembolism among surgical patients treated in Department of Veterans Affairs hospitals, 2005-2010. Thromb Res 2015;135(04):636-642 
29 Alsubaie H, Leggett C, Lambert P, et al. Diagnosis of VTE postdischarge for major abdominal and pelvic oncologic surgery: implications for a change in practice. Can J Surg 2015;58(05):305-311

30 Bouras G, Burns EM, Howell AM, Bottle A, Athanasiou T, Darzi A. Risk of post-discharge venous thromboembolism and associated mortality in general surgery: a population-based cohort study using linked hospital and primary care data in England. PLoS One 2015;10(12):e0145759

31 Silverstein MD, Heit JA, Mohr DN, Petterson TM, O'Fallon WM, Melton LJ III. Trends in the incidence of deep vein thrombosis and pulmonary embolism: a 25-year population-based study. Arch Intern Med 1998;158(06):585-593 